\title{
Proposta de emprego de conceitos de equilíbrio químico na descrição matemática da câmara de combustão de caldeira siderúrgica
}

\author{
Turetta, L. F..$^{*}$; Costa, A. O. S. ${ }^{2}$ \\ 1 Mestranda do Programa de Pós-Graduação em Engenharia Química, Universidade Federal do Espírito Santo, Alegre, \\ ES, Brasil. \\ 2 Professora do Programa de Pós-Graduação em Engenharia Química, Universidade Federal do Espírito Santo, \\ Alegre, ES, Brasil. \\ *e-mail: leticialft@hotmail.com
}

\begin{abstract}
Resumo
A queima de um combustível na câmara de combustão na presença de ar gera vapor, cinzas e gases de exaustão. A determinação da composição de saída das cinzas e dos gases de exaustão é de extrema importância ambiental e econômica para uma empresa. A descrição fenomenológica deste processo pode ser realizada empregando-se diferentes técnicas. Na modelagem matemática da câmara de reação de combustão utilizando a metodologia cinética é necessário: descrever as reações de combustão do combustível, determinar as taxas das reações, determinar os valores dos parâmetros cinéticos envolvidos e resolver o balanço de massa. Uma alternativa a esta técnica pode ser o emprego da minimização da energia livre de Gibbs do sistema. A energia livre de Gibbs total de um sistema fechado à temperatura e a pressão constantes devem diminuir durante um processo irreversível e a condição de equilíbrio é atingida quando a energia de Gibbs alcança seu valor mínimo. Portanto propondo um problema de otimização onde a energia livre de Gibbs é minimizada junto às restrições mássicas, pôde-se determinar a composição de saída de um sistema. O objetivo deste estudo consistiu em analisar as duas metodologias citadas anteriormente, e fazer uma descrição matemática da câmara de combustão de uma caldeira siderúrgica.
\end{abstract}

\begin{abstract}
The burning of a fuel in the furnace produces steam, ash and exhaustion gases. The determination of the ash and exit gas composition is important by environmental and economic issues to the industry. The phenomenological description of this process can be done by different techniques. To model the furnace by the kinetic methodology is needed to know the fuel combustion reactions, to determine the rates of these reactions, the kinetic parameters values and solve the mass balance. An alternative proposal to this technique can be to use minimization of the free Gibbs energy. The total Gibbs free energy of a closed system at constant temperature and pressure decreases during an irreversible process and the equilibrium condition is reached when the Gibbs energy reaches its minimum value. Therefore, in this case, it is proposed an optimization problem where the Gibbs free energy is minimized considering the mass restrictions. The obtained result informs the composition of the system. The goal of this study is to analyze the two methods previously mentioned aiming the mathematical description of the furnace of a steel boiler.
\end{abstract}

Keywords (Palavras chaves): Composição de saída, Mecanismo Cinético, Energia Livre de Gibbs. 


\section{Introdução}

A indústria siderúrgica é uma grande consumidora de energia. Este setor em 2013 consumiu cerca de $26 \%$ da energia elétrica industrial disponível no Brasil [1]. A produção de aço é um forte indicador do estágio de desenvolvimento econômico e seu consumo cresce ao longo dos anos. Durante o processo de produção de ferro gusa e aço quatro tipos de combustíveis são gerados: gás de alto forno, gás de coqueria, gás de aciaria e alcatrão. Esses quatro subprodutos apresentam elevada capacidade de geração de energia. $\mathrm{Na}$ usina siderúrgica, esses subprodutos são direcionados às centrais termoelétricas. A central termoelétrica é uma instalação industrial que transforma a energia térmica obtida a partir da queima de combustíveis nas caldeiras em energia elétrica. A queima da mistura desses subprodutos na presença de ar gera vapor e gases de exaustão que saem nas chaminés. A determinação da composição de saída desses gases é de extrema importância ambiental e econômica para uma empresa. Todavia, o conhecimento desta composição nem sempre se mostra trivial. O objetivo deste estudo consiste em analisar duas diferentes metodologias para a descrição matemática da câmara de combustão de uma caldeira siderúrgica: descrição empregando modelos cinéticos e descrição empregando a minimização da energia livre de Gibbs.

\section{Metodologias}

\subsection{Metodologia cinética}

Para modelar uma fornalha de uma caldeira utilizando uma abordagem cinética é necessário conhecer a cinética das reações envolvidas na combustão do combustível. Na literatura, diversas pesquisas usam esta metodologia para predizer a composição do sistema para a queima de diferentes combustíveis [2-7]. A cinética é definida como a ciência que estuda a velocidade das reações químicas dos processos e os fatores que as influenciam. Existem muitos fatores que influenciam as reações químicas. Na modelagem do sistema, a temperatura, a pressão, o uso de catalisadores, formação de inibidores e a superfície de contato do combustível podem ser considerados importantes para o modelo [8].

$\mathrm{Na}$ modelagem de uma fornalha empregando abordagem cinética é necessário conhecer as reações de formação de todos os produtos envolvidos. As reações de combustão possuem equações de taxas que descrevem o consumo do combustível e a formação dos produtos. As equações de taxa possuem, além das concentrações, as constantes cinéticas. Tais constantes são obtidas empiricamente e podem ser obtidas da literatura. Todavia, tais parâmetros são específicos para uma dada reação em uma dada condição operacional. Também é possível propor novas equações de taxas com novas constantes cinéticas, mas para isso se faz necessário determinar todos os parâmetros cinéticos de interesse por métodos experimentais.

$\mathrm{Na}$ modelagem da combustão do carvão pulverizado em uma câmara de reação são descritos 7 mecanismos de reação, com suas respectivas equações de taxas e parâmetros [5]. Uma pesquisa desenvolvida com objetivo modelar a combustão do carbonato de dimetilo utiliza-se 25 mecanismos diferentes de reação com suas respectivas equações de taxas e parâmetros [4]. As caldeiras da central termoelétrica de uma usina siderurgia operam com um mix (combinação) de combustível como mencionado anteriormente. Esse mix de combustível é composto usualmente pelos gases de alto forno, coqueria e aciaria além do alcatrão, totalizando mais de 37 compostos diferentes, sendo 25 somente do alcatrão. Neste caso a metodologia cinética pode não ser recomendada devido ao grande volume de reações e espécies químicas envolvidas no processo.

\subsection{Metodologia da minimização da energia livre de Gibbs}

Uma alternativa à metodologia cinética é a metodologia que emprega a metodologia da minimização da energia livre de Gibbs. $\mathrm{Na}$ literatura diversas pesquisas usam esta metodologia para predizer a composição de sistemas com muitas espécies e diversas fases em equilíbrio químico [9-14].

A energia de Gibbs total de um sistema fechado, à temperatura e a pressão constantes, diminui durante um processo irreversível e a condição de equilíbrio é atingida quando a energia de Gibbs alcança seu valor mínimo [15].

Em um sistema fechado com $F$ fases e $N$ diferentes espécies químicas, a energia livre de Gibbs é calculada por uma combinação dos potenciais químicos de cada componente em cada fase, expressa pela Equação (1) [12].

$$
G=\sum_{j=1}^{F} \sum_{i=1}^{N} \mu_{i, j} \cdot n_{i, j}
$$

Onde,

G - energia livre de Gibbs total do sistema fechado; 
$\mu_{\mathrm{i}, \mathrm{j}}$ - potencial químico do componente i na fase j; $\mathrm{n}_{\mathrm{i}, \mathrm{j}}$ - número de mols do componente i na fase $\mathrm{j}$.

O potencial químico da espécie $\mathrm{i}$ na fase j é descrita pela Equação (2).

$$
\mu_{i, j}=G_{f_{i, j}}^{\circ}+R \cdot T \cdot \ln \left(\frac{f_{i, j}}{f_{i, j}^{\circ}}\right)
$$

Onde,

$G_{\mathrm{fi}, \mathrm{j}}^{\circ}$ - energia livre de Gibbs de formação do componente i na fase j;

$R$ - constante universal dos gases;

$T$ - temperatura de operação da fornalha;

$f_{\mathrm{i}, \mathrm{j}}$ - fugacidade do componente i na fase $\mathrm{j}$;

$f_{\mathrm{i}, \mathrm{j}}^{\circ}$ - fugacidade do componente i na fase $\mathrm{j}$ no estado padrão;

$P$ - pressão do sistema.

Se considerarmos que as fases presentes no sistema se comportam idealmente temos a Equação 3. Desta forma, a função objetivo (Equação 1) para um sistema gasoso ideal pode ser descrita como na Equação 4 [12].

$$
\begin{gathered}
\frac{f_{i, j}}{f_{i, j}^{\circ}}=P \cdot \frac{n_{i, j}}{\sum n_{i, j}} \\
G=\sum_{j=1}^{F} \sum_{i=1}^{N}\left(G_{f_{i, j}}^{\circ}+R \cdot T \cdot \ln \left(P \cdot \frac{n_{i, j}}{\sum n_{i, j}}\right)\right) \cdot n_{i}
\end{gathered}
$$

Para determinar a energia livre de Gibbs de formação do componente $i$ na fase $j$ se faz necessário obter os valores da energia livre de Gibbs de formação dos produtos e reagentes (Equação 5). A energia livre de Gibbs de formação dos produtos é determinada pela entalpia de formação padrão $\left(H^{\circ}\right)$, entropia padrão de formação $\left(\mathrm{S}^{\circ}\right)$ e das capacidades caloríficas do componente $i$ na fase $j\left(\mathrm{C}_{\mathrm{p}}\right)$ como explicitado na Equação 6 [16].

$$
\begin{aligned}
& G_{f_{i, j}}^{\circ}= G_{f_{\text {produtos }}}-G_{f_{\text {reagentes }}} \\
& G_{f_{\text {produtos }}=}=\left(H^{\circ}+\int_{T_{\text {ref }}}^{T} C p d T\right)-T \\
& \cdot\left(S^{\circ}+\int_{T_{\text {ref }}}^{T} \frac{C p d T}{T}\right)
\end{aligned}
$$

Além disso, como se trata de um sistema fechado, as equações que descrevem as relações mássicas do sistema devem ser respeitadas durante a resolução do problema de minimização. Isto é, a solução do problema de otimização deve respeitar o princípio da conservação da massa. Assim, para a completa apresentação do problema de otimização se faz necessário explicitar relações matemáticas que descrevem restrições de igualdade.

Para a proposta das restrições de igualdade, considera-se que a composição química elementar do meio reacional é constante para um sistema fechado. Ou seja, não ocorrem reações nucleares, de forma que os elementos químicos estarão distribuídos entre as substâncias químicas, mas obedecendo a uma concentração química elementar fixa. Portanto, para cada elemento químico $k$ a restrição de igualdade é expressa pela Equação [12].

$$
\begin{gathered}
b_{k}=\sum_{\substack{j=1 \\
k=1,2, \ldots, M}}^{F} a_{i=1} \cdot n_{i, j} \\
\mathrm{n}_{\mathrm{i}, \mathrm{j}} \geq 0
\end{gathered}
$$

Onde,

$b_{k}$ - número total de átomos do k-ésimo elemento da mistura reacional;

$a_{k, i}$ - coeficiente estequiométrico do elemento $\mathrm{k}$ na espécie química $i$.

Diante do exposto, é possível determinar a composição dos gases de exaustão resolvendo o problema de otimização composto pela função objetivo que será minimizada é a Equação 4, sujeito a dois conjuntos distintos de restrições. $O$ primeiro conjunto de restrições de igualdade (Equação 7), uma para cada elemento químico presente no meio reacional. O segundo conjunto de restrições (Equação 8) impede que número de mols do componente seja negativo o que fisicamente não faz sentido.

\section{Resultados e discussão}

A Tabela 1 reúne oito itens importantes ao se determinar qual a melhor metodologia aplicada na modelagem de uma caldeira siderúrgica, quando a finalidade é prever a composição de saída dos gases de exaustão.

Em relação à Tabela 1:

A - Quando a metodologia cinética é escolhida para a modelagem do sistema é necessário conhecer todas as reações que ocorrem com o combustível. A segunda metodologia não exige o conhecimento prévio das reações. Ressalta-se que o processo de queima de um combustível pode envolver reações químicas complexas e/ou desconhecidas. Para a aplicação da segunda metodologia, o que necessita ser informado é uma previsão de quais substâncias serão formadas. $O$ termo previsão é utilizado, pois se alguma substância informada (prevista) não compor a composição de saída, o algoritmo de otimização irá informar zero para a concentração desta substância no sistema. 
Tabela 1: Comparação do uso da metodologia cinética com a metodologia da minimização da energia livre de Gibbs.

\begin{tabular}{lcc}
\multicolumn{1}{c}{ Itens } & $\begin{array}{c}\text { Metodologia } \\
\text { cinética }\end{array}$ & $\begin{array}{c}\text { Minimização da } \\
\text { energia livre de } \\
\text { Gibbs }\end{array}$ \\
\hline $\begin{array}{l}\text { A- Determinar as reações químicas do processo } \\
\text { B- Determinar fatores que influenciam as reações } \\
\text { químicas }\end{array}$ & Sim & Não \\
C- Determinar as equações de taxa de reação & Sim & Não \\
química & Sim & Não \\
D- Determinação de parâmetros cinéticos & Sim & Não \\
E- Limitar faixa de temperatura de operação & Sim & Não \\
F- Flexibilidade na mudança do estado físico do & Não & Sim \\
combustível & Baixa & Alta \\
G- Flexibilidade na mudança de combustível & Baixa & Sim \\
H- Aplicação de informações termodinâmicas & & \\
\hline
\end{tabular}

B - Como já mencionado existem diversos fatores que influenciam as reações químicas. $\mathrm{Na}$ metodologia cinética, esses fatores podem ser incorporados nos modelos matemáticos. Portanto nessa metodologia, fatores como o uso de catalisadores e formação de inibidores, podem ser objeto de estudo para a otimização de um processo industrial. Na segunda metodologia não existe essa possibilidade, pois a Energia livre de Gibbs não contempla a influência desses fatores.

C - Na metodologia cinética é possível propor novas equações de taxas que contabilizem as influências de diversos fatores nas reações. Muitos pesquisadores utilizam as equações de taxa da literatura ou propõem novas equações. A segunda metodologia as equações de taxa não são aplicadas.

$D$ - As equações da metodologia cinética na sua grande maioria possuem parâmetros. Esses parâmetros são determinados experimentalmente. O uso de equações e parâmetros já existentes na literatura é comum. Entretanto, quando o sistema a ser estudado se diverge em muitos aspectos do sistema gerador dos parâmetros pode ocorrer a geração de desvios do modelo ideal. Na segunda metodologia não se utiliza parâmetros cinéticos na modelagem.

E - Na metodologia cinética a determinação da temperatura de operação deve ser avaliada antes do início da modelagem. Diversos parâmetros são determinados considerando uma faixa de temperatura. $\mathrm{Na}$ segunda metodologia a variação da temperatura pode ser objeto de análise, pois em muitos sistemas como o de combustão a temperatura de operação é um dos fatores que influenciam o processo. Na metodologia baseada na minimização da energia livre de Gibbs, a temperatura de operação tem que ser informada e pode ser simulada em qualquer faixa de temperatura desejada pelo usuário. Em alguns processos não existem a possibilidade de medir a temperatura de operação, isso pode gerar alguns desvios na simulação.

F - A mudança do estado físico do combustível é uma das informações requeridas na modelagem cinética, pois essa variação influência na escolha da equação de taxa da reação. A superfície de contato do combustível é um dos diversos fatores abordados pela cinética o que não é possível quando se utiliza a energia de Gibbs. A segunda metodologia permite a simulação de um combustível sólido líquido e/ou gasoso.

G - A mudança de combustível ou a mudança da composição do combustível de uma caldeira é uma manobra muito comum na indústria. $\mathrm{Na}$ metodologia cinética, a consideração de tais alterações podem refletir em alterações drásticas no modelo o que pode tornar inviável tal análise. $\mathrm{Na}$ segunda metodologia, a mudança de combustível não impede a aplicação do modelo, pois a informação requerida pelo modelo é a vazão atômica do combustível, ou seja, o usuário precisa informar a composição química elementar do combustível. Assim, neste caso, alterações na composição química do combustível podem ser incorporadas sem que seja necessária uma alteração drástica na rotina de cálculo.

$\mathrm{H}$ - Na aplicação da metodologia cinética pode ser necessário incorporar à rotina de cálculo conceitos da primeira lei da termodinâmica (balanço de energia). $\quad \mathrm{Na}$ metodologia da minimização da energia livre de Gibbs se faz necessário a obtenção de dados termodinâmicos como: a entalpia de formação padrão, entropia padrão de formação e das capacidades caloríficas dos componentes em cada fase

\section{Conclusão}

Neste trabalho foram apresentadas duas metodologias diferentes que podem ser empregadas para prever a composição de saída dos gases de exaustão em uma usina siderúrgica. 
A metodologia cinética mostra-se eficiente, quando - combustível utilizado, não esta sujeito a variações de composição química e física. A metodologia da minimização da energia livre de Gibbs pode ser aplicada a um processo de queima de um combustível que envolve reações químicas complexas e desconhecidas. Esta segunda metodologia é flexível quanto a mudanças na composição dos combustíveis e mostra-se eficiente, quando se deseja analisar variações de temperatura e excesso de oxigênio. As variações na temperatura e na vazão de oxigênio na queima do combustível em uma caldeira são informações valiosas para a indústria, pois a eficiência térmica de uma caldeira esta atrelada a essas variáveis.

Portanto, as duas metodologias podem ser aplicadas para prever a concentração de equilíbrio das espécies químicas presentes na fornalha de uma caldeira siderúrgica.

\section{Agradecimento}

Os autores agradecem à CAPES pela bolsa de mestrado e à FAPES pela bolsa pesquisador capixaba.

\section{Referências}

[1] EPE. Demanda de energia 2050. EPE. Rio de Janeiro, p. 245. 2014.

[2] LÓPEZ, I.; PASSEGGI, M.; BORZACCONI, L. Variable kinetic approach to modelling an industrial waste anaerobic digester. Biochemical

Engineering Journal, v. 96, p. 7-13, 2015.

[3] MURCIA, M. D. et al. Development of a kinetic model for the UV/ $\mathrm{H}_{2} \mathrm{O}_{2}$ photodegradation of 2,4dichlorophenoxiacetic acid. Chemical Engineering Journal, v. 266, p. 356-367, 2015.

[4] GLAUDE, P. A.; PITZ, W. J.; THOMSON, M. J. Chemical kinetic modeling of dimethyl carbonate in an opposed-flow diffusion flame. Proceedings of the Combustion Institute, v. 30, p. 1111-1118, 2005.

[5] RODRIGUES, C. P. et al. SIMULATION OF PULVERIZED COAL FIRED BOILER:REACTION CHAMBER. Thermal Engineering, v. 4, p. 61-68, 2005.

[6] RAGGI, M. V. K. MODELAGEM DA CINÉTICA QUÍMICA DE FORMAÇÃO DE NOX E CO EM
MOTORES COM IGNIÇÃO POR CENTELHA. 130

f. Dissertação (Mestrado em Engenharia

Mecânica) - Programa de Pós-Graduação em Engenharia mecânica, Pontifícia Universidade Católica de Minas Gerais. 2005.

[7] JÚNIOR, F. S. D. F. MODELAGEM E SIMULAÇÃO DINÂMICA DE UM COMBUSTOR DE GÁS NATURAL EM LEITO FLUIDIZADO. 118

f. Dissertação (Mestrado em Engenharia Química)

- Programa de Pós-Graduação em Engenharia Química, Universidade federal de Pernanbuco. 2004.

[8] LEVENSPIEL, O. Engenharia das reação Químicas. 3. ed. São Paulo: Blucher, v. 1, 2000.

[9] SUNDMAN, B.; LUB, X.-G.; OHTANI, H. The implementation of an algorithm to calculate thermodynamic equilibria for multi-component systems with non-ideal phases in a free software. Computational Materials Science, v. 101, p. 127-137, 2015.

[10] KANGAS, P.; HANNULA, I.; KOUKKARI, P. Modelling super-equilibrium in biomass gasification with the constrained Gibbs energy method. Fuel, v. 129, p. 86-94, 2014.

[11] NÉRON, A.; LANTAGNE, G.; MARCOS, B. Computation of complex and constrained equilibria by minimization of the Gibbs free energy.

Chemical Engineering Science, v. 82, p. 260271, 2012.

[12] SILVA, W. L.; RIBEIRO, J. C. T.; COSTA, E. F. D. J. Reduction efficiency prediction of CENIBRA's recovery boiler by direct minimization of gibbs free energy. Brazilian Journal of Chemical

Engineering, v. 25, p. 603 - 611, 2008.

[13] FOURNEL, S. et al. Predicting gaseous emissions from small-scale combustion of agricultural biomass fuels. Bioresource Technology, v. 179, p. 165-172, 2014.

[14] S.COSTA, A. O.; BISCAIA, E. C.; LIMA, E. L. Chemical Composition Determination at the Bottom Region of a Recovery Boiler Furnace by Direct Minimization of Gibbs Free Energy. Canadian Journal of Chemical Engineering, v. 83, p. 1-8, 2005.

[15] SMITH, J. M.; VAN NESS, H. C.; ABBOTT, M. $M$. Introdução à termodinâmica da engenharia química. 5. ed. Rio de Janeiro: JC, v. 1, 2000. 